\title{
Nuclear dependence of charm production
}

\section{The SELEX Collaboration}

A. Blanco-Covarrubias ${ }^{11}$, J. Engelfried ${ }^{11, a}$, U. Akgun ${ }^{13}$, G. Alkhazov ${ }^{9}$, J. Amaro-Reyes ${ }^{11}$, A.G. Atamantchouk ${ }^{9, b}$, A.S. Ayan $^{13}$, M.Y. Balatz ${ }^{6, b}$, N.F. Bondar ${ }^{9}$, P.S. Cooper ${ }^{4}$, L.J. Dauwe ${ }^{14, b}$, G.V. Davidenko ${ }^{6}$, U. Dersch ${ }^{7, c}$, A.G. Dolgolenko ${ }^{6}$, G.B. Dzyubenko ${ }^{6}$, R. Edelstein ${ }^{2}$, L. Emediato $^{16}$, A.M.F. Endler ${ }^{3}$, I. Eschrich ${ }^{7, d}$, C.O. Escobar ${ }^{16, \mathrm{e}}$, N. Estrada ${ }^{11}$, A.V. Evdokimov ${ }^{6}$, I.S. Filimonov ${ }^{8, b}$, A. Flores-Castillo ${ }^{11}$, F.G. Garcia ${ }^{16,4}$, V.L. Golovtsov ${ }^{9}$ ，P. Gouffon ${ }^{16}$, E. Gülmez ${ }^{1}$ ，M. Iori ${ }^{15}$ ， S.Y. Jun ${ }^{2}$ ，M. Kaya ${ }^{13, f}$ ，J. Kilmer ${ }^{4}$ ，V.T. Kim ${ }^{9}$, L.M. Kochenda ${ }^{9}$, I. Konorov ${ }^{7, g}$, A.P. Kozhevnikov ${ }^{5}$, A.G. Krivshich ${ }^{9}$, H. Krüger ${ }^{7, h}$, M.A. Kubantsev ${ }^{6}$, V.P. Kubarovsky ${ }^{5}$, A.I. Kulyavtsev ${ }^{2,4}$, N.P. Kuropatkin ${ }^{9,4}$, V.F. Kurshetsov ${ }^{5}$, A. Kushnirenko ${ }^{2,5}$, J. Lach ${ }^{4}$, L.G. Landsberg ${ }^{5, \mathrm{~b}}$, I. Larin ${ }^{6}$, E.M. Leikin ${ }^{8}$, G. López-Hinojosa ${ }^{11}$, T. Lungov ${ }^{16}$, V.P. Maleev ${ }^{9}$, D. Mao ${ }^{2, i}$, P. Mathew ${ }^{2, j}$, M. Mattson ${ }^{2}$, V. Matveev ${ }^{6}$, E. McCliment ${ }^{13}$, M.A. Moinester ${ }^{10}$, V.V. Molchanov ${ }^{5}$ A. Morelos ${ }^{11}$, A.V. Nemitkin ${ }^{8}$, P.V. Neoustroev ${ }^{9}$, C. Newsom ${ }^{13}$, A.P. Nilov ${ }^{6, b}$, S.B. Nurushev ${ }^{5}$, A. Ocherashvili ${ }^{10, k}$, Y. Onel ${ }^{13}$, S. Ozkorucuklu ${ }^{13,1}$, A. Penzo ${ }^{17}$, S.V. Petrenko ${ }^{5}$, M. Procario ${ }^{2, \mathrm{~m}}$, V.A. Prutskoi ${ }^{6}$, B.V. Razmyslovich ${ }^{9, \mathrm{n}}$, V.I. Rud ${ }^{8}$, J. Russ ${ }^{2}$, J.L. Sánchez-López ${ }^{11}$ ，J. Simon ${ }^{7, o}$, A.I. Sitnikov ${ }^{6}$, V.J. Smith ${ }^{12}$, M. Srivastava ${ }^{16}$, V. Steiner $^{10}$, V. Stepanov ${ }^{9, n}$, L. Stutte ${ }^{4}$, M. Svoiski ${ }^{9, n}$, N.K. Terentyev ${ }^{9,2}$, I. Torres ${ }^{11, p}$, L.N. Uvarov ${ }^{9}$, A.N. Vasiliev ${ }^{5}$, D.V. Vavilov ${ }^{5}$, E. Vázquez-Jáuregui ${ }^{11}$, V.S. Verebryusov ${ }^{6}$, V.A. Victorov ${ }^{5}$, V.E. Vishnyakov $^{6}$, A.A. Vorobyov $^{9}$, K. Vorwalter ${ }^{7, q}$, J. You ${ }^{2,4}$, R. Zukanovich-Funchal ${ }^{16}$

${ }^{1}$ Bogazici University, 80815 Bebek, Istanbul, Turkey

${ }^{2}$ Carnegie-Mellon University, Pittsburgh, PA 15213, USA

${ }^{3}$ Centro Brasileiro de Pesquisas Físicas, Rio de Janeiro, Brazil

${ }^{4}$ Fermi National Accelerator Laboratory, Batavia, IL 60510, USA

${ }^{5}$ Institute for High Energy Physics, Protvino, Russia

${ }^{6}$ Institute of Theoretical and Experimental Physics, Moscow, Russia

${ }^{7}$ Max-Planck-Institut für Kernphysik, 69117 Heidelberg, Germany

${ }^{8}$ Moscow State University, Moscow, Russia

${ }^{9}$ Petersburg Nuclear Physics Institute, St. Petersburg, Russia

${ }^{10}$ Tel Aviv University, 69978 Ramat Aviv, Israel

${ }^{11}$ Instituto de Fisica, Universidad Autónoma de San Luis Potosí, Manuel Nova \#6, Zona Universitaria, San Luis Potosí 78246, Mexico

${ }^{12}$ University of Bristol, Bristol BS8 1TL, UK

${ }^{13}$ University of Iowa, Iowa City, IA 52242, USA

${ }_{15}^{14}$ University of Michigan-Flint, Flint, MI 48502, USA

${ }^{15}$ University of Rome "La Sapienza" and INFN, Rome, Italy

${ }^{16}$ University of São Paulo, São Paulo, Brazil

${ }^{17}$ University of Trieste and INFN, Trieste, Italy

Received: 22 July 2009 / Revised: 30 September 2009 / Published online: 20 October 2009 (C) Springer-Verlag / Società Italiana di Fisica 2009

a e-mail: jurgen@ifisica.uaslp.mx

${ }^{\mathrm{b}}$ Deceased

${ }^{\mathrm{c}}$ Present address: Advanced Mask Technology Center, Dresden, Germany

${ }^{\mathrm{d}}$ Present address: University of California at Irvine, Irvine, CA 92697 , USA

${ }^{\text {e}}$ Present address: Instituto de Física da Universidade Estadual de Campinas, UNICAMP, San Paulo, Brazil

${ }^{f}$ Present address: Kafkas University, Kars, Turkey

gPresent address: Physik-Department, Technische Universität München, 85748 Garching, Germany

${ }^{\text {h}}$ Present address: The Boston Consulting Group, München, Germany

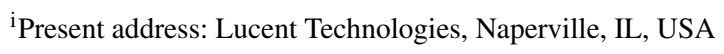

jPresent address: Baxter Healthcare, Round Lake, IL, USA

${ }^{k}$ Present address: NRCN, 84190 Beer-Sheva, Israel

${ }^{1}$ Present address: Süleyman Demirel Universitesi, Isparta, Turkey

${ }^{m}$ Present address: DOE, Germantown, MD, USA

${ }^{n}$ Present address: Solidum, Ottawa, Ontario, Canada

${ }^{\circ}$ Present address: Siemens Healthcare, Erlangen, Germany

pPresent address: Benemerita Universidad Autónoma de Puebla (BUAP), Mexico, Mexico

qPresent address: Allianz Insurance Group IT, München, Germany 
Abstract Using data taken by SELEX during the 19961997 fixed target run at Fermilab, we study the production of charmed hadrons on copper and carbon targets with $\Sigma^{-}, p$, $\pi^{-}$, and $\pi^{+}$beams. Parametrizing the dependence of the inclusive production cross section on the atomic number $A$ as $A^{\alpha}$, we determine $\alpha$ for $D^{+}, D^{0}, D_{s}^{+}, D^{+}(2010), \Lambda_{c}^{+}$, and their respective anti-particles, as a function of their transverse momentum $p_{t}$ and scaled longitudinal momentum $x_{F}$. Within our statistics there is no dependence of $\alpha$ on $x_{F}$ for any charm species for the interval $0.1<x_{F}<1.0$. The average value of $\alpha$ for charm production by pion beams is $\alpha_{\text {meson }}=0.850 \pm 0.028$. This is somewhat larger than the corresponding average $\alpha_{\text {baryon }}=0.755 \pm 0.016$ for charm production by baryon beams $\left(\Sigma^{-}, p\right)$.

PACS 13.85.Ni $\cdot$ 14.65.Dw $\cdot 24.85 .+\mathrm{p}$

\section{Introduction}

The inclusive production of various outgoing hadrons from a specific incident beam particle interacting in a nuclear target has been studied for many years. The usual characterization of the process is that in a complex nucleus of atomic number $A$, the single nucleon production cross section is increased by a factor $A^{\alpha}$. If the outgoing particle is absorbed with the same cross section as the incoming beam particle, then $\alpha \sim 2 / 3$. Conversely, production of heavy quarks by hard scattering with slow hadronization may lead to minimal absorption and give $\alpha \sim 1$.

Experiments have used a range of techniques to determine $\alpha$ for a wide selection of charm and strange hadrons. Some experiments have studied generic charm hadron production by detecting only muons from semi-leptonic decays [1-3]. Others have measured $\alpha$ for hidden charm in $J / \psi$ production [4-6]. A third group have looked at specific charm or strange final state hadrons [7-17]. Until this experiment, no single experiment covered the whole family of charm hadrons over a wide range of kinematic variables $p_{t}$ and $x_{F}$, using $\pi^{+}, \pi^{-}, p$, and $\Sigma^{-}$beams. For the first time these new data allow one to look for systematic variations of $\alpha$ for different kinematic regions, different groups of outgoing charm hadrons, or different beam hadrons. This broad data set is important for comparing to the variety of theoretical models that have been proposed.

The production of strange particles with a proton beam shows [7,8] a strong dependence of $\alpha$ on both $x_{F}$ and $p_{t}$, with $\alpha<0.5$ in some bins of $\left(x_{F}, p_{t}\right)$. Open charm production was measured [9-17] for pion, proton, and neutron beams, in different ranges of $x_{F}$, for $D^{ \pm}, D^{0}, D^{*}$, and $D_{s}$ mesons as well as the $\Lambda_{c}^{+}$, but most experiments only had one beam particle and/or final state hadron, or averaged over several mesons, publishing just one value for $\alpha$. In addition to the previously mentioned muon measurements [1-6], prompt neutrino production assumed to be from charm decays is reported in [18]. As summarized in [19, 20], many measurements of open charm production concentrate in the central region $x_{F} \sim 0$ and find $\alpha \sim 1$ for the specific final states measured. For charmonium production or muontriggered inclusive charm production at large $x_{F}, \alpha$ decreases, approaching $2 / 3$.

Studying the basic production and suppression mechanisms in charm hadroproduction is important for understanding non-perturbative aspects of heavy hadron production. These results also impact other fields like Heavy-Ion collisions (see a review [21]) and Cosmic Ray Physics, where air shower Monte Carlo simulations have to take into account the production of charm particles.

We present in this letter a new measurement for $\alpha$ in the range of $0.1<x_{F}<1$, for 14 different open charm particles and decay modes, produced by four different beam particles. In any single beam/charm-particle combination this experiment has statistics that are similar to or better than those from any other previous measurement.

\section{Experimental apparatus}

The experimental setup of the SELEX experiment is described elsewhere [22]. We point out the most important features of the setup used in this analysis. SELEX is a 3-stage magnetic spectrometer, designed for high acceptance forward $\left(x_{F} \gtrsim 0.1\right)$ interactions. $600 \mathrm{GeV} / c$ negative $(\simeq 50 \%$ $\left.\Sigma^{-}, \simeq 50 \% \pi^{-}\right)$and $540 \mathrm{GeV} / c$ positive beam particles $\left(\simeq 92 \% p, \simeq 8 \% \pi^{+}\right)$, interact in five target foils, described in Table 1 . The physical properties of the target foils were measured before the installation into the experimental setup, and the thicknesses and positions were verified by measuring the positions of the primary vertices. Every beam particle is individually tagged by a Transition Radiation Detector, and the meson (baryon) contamination in the baryon (meson) beam is below $1 \%$.

The spectrometer had silicon strip detectors to measure the incoming beam and outgoing tracks. Momenta of particles deflected by the analyzing magnets were measured by a system of proportional wire chambers (PWCs), drift chambers and silicon strip detectors. Momentum resolution for a typical $100 \mathrm{GeV} / c$ track was $\sigma_{p} / p \approx 0.5 \%$. Charged particle identification was performed with a Ring Imaging Cherenkov detector (RICH) [23], which distinguished $K^{ \pm}$from $\pi^{ \pm}$up to $165 \mathrm{GeV} / c$. The proton identification efficiency was $>95 \%$ above proton threshold $(\approx 90 \mathrm{GeV} / c)$. For pions reaching the RICH detector, the total mis-identification probability due to all sources of confusion was $<4 \%$.

Interactions in the five target foils were selected by a scintillator trigger. The trigger for charm required at least four 
Table 1 Physical properties of materials in the charm production targets region. The layout is shown in Fig. 1

\begin{tabular}{lllllll}
\hline Name & Material & $\begin{array}{l}\text { Thickness } L \\
{[\mathrm{~cm}]}\end{array}$ & $\begin{array}{l}\text { Position } \\
{[\mathrm{cm}]}\end{array}$ & $A$ & $\begin{array}{l}\text { Density } \rho \\
{\left[\mathrm{g} / \mathrm{cm}^{3}\right]}\end{array}$ & $\begin{array}{l}\lambda_{\text {int }} \\
{[\%]}\end{array}$ \\
\hline S4 & Scintillator & 0.158 & 7.27 & - & 1.03 & 0.20 \\
6 & Copper & 0.159 & 6.13 & 63.5 & 8.96 & 1.06 \\
7 & Copper & 0.119 & 4.62 & 63.5 & 8.96 & 0.76 \\
8 & Diamond & 0.220 & 3.10 & 12 & 3.25 & 0.82 \\
9 & Diamond & 0.220 & 1.61 & 12 & 3.25 & 0.82 \\
10 & Diamond & 0.220 & 0.11 & 12 & 3.25 & 0.82 \\
IC1 & Scintillator & 0.200 & 2.46 & - & 1.03 & 0.25 \\
IC2 & Scintillator & 0.200 & 2.97 & - & 1.03 & 0.25 \\
\hline
\end{tabular}

Fig. 1 Scale drawing of the charm production target region. In addition to the five targets (2 Copper, 3 Diamond) we also indicate the location of some of the scintillators used in the trigger (S4, IC1, IC2) and the first two planes $(1 \mathrm{x}, 1 \mathrm{y}$, from 20 in total) of the silicon strip detectors. The physical properties of the elements are shown in Table 1

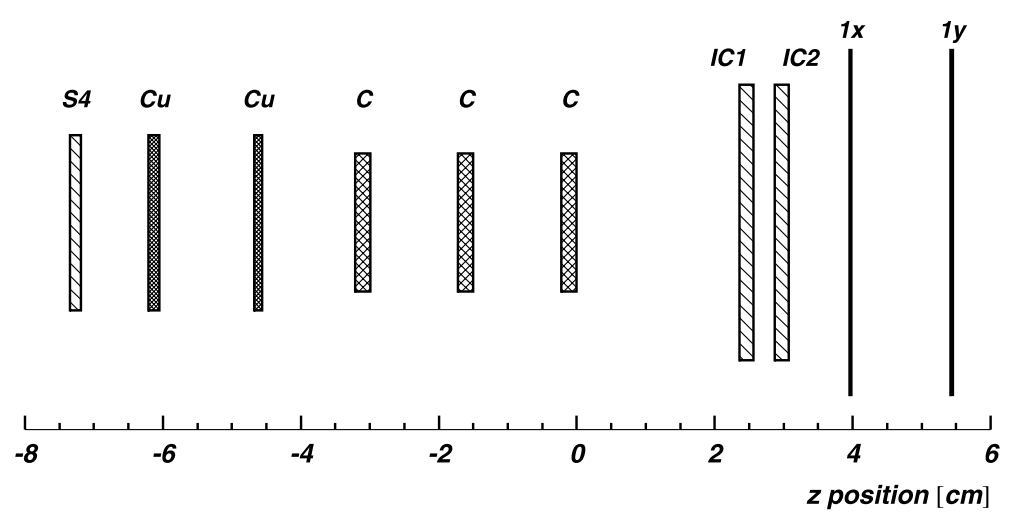

charged tracks downstream of the targets as indicated by an interaction counter (IC1, IC2, see Fig. 1), no signal in a veto counter (S4) upstream of the targets, and at least 2 hits in a scintillator hodoscope after the second analyzing magnet. It accepted about $1 / 3$ of all inelastic interactions. Triggered events were further tested in an on-line computational filter based on downstream tracking and particle identification information. The on-line filter selected events that had evidence of a secondary vertex from tracks completely reconstructed using the forward PWC spectrometer and the vertex silicon. This filter reduced the data size by a factor of nearly 8 at a cost of about a factor of 2 in charm yield. From a total of $15.2 \times 10^{9}$ interactions during the 1996-1997 fixed target run about $10^{9}$ events were written to tape.

\section{Data analysis}

To determine the charm production cross section dependence on the nuclear mass $A$, we have to determine the number of charm particles produced in any single target, and take into account the number of nuclei in the Carbon and Copper targets. Parametrizing the cross section $\propto A^{\alpha}$, we obtain

$\alpha=\frac{\ln \left(\frac{N_{\mathrm{Cu}}}{N_{\mathrm{C}}} \frac{\rho_{\mathrm{C}}}{\rho_{\mathrm{Cu}}} \frac{L_{\mathrm{C}}}{L_{\mathrm{Cu}}} \frac{A_{\mathrm{Cu}}}{A_{\mathrm{C}}}\right)}{\ln \left(\frac{A_{\mathrm{Cu}}}{A_{\mathrm{C}}}\right)}=\frac{\ln \frac{N_{\mathrm{Cu}}}{N_{\mathrm{C}}}}{\ln \frac{A_{\mathrm{Cu}}}{A_{\mathrm{C}}}}+\frac{\ln \left(\frac{\rho_{\mathrm{C}}}{\rho_{\mathrm{Cu}}} \frac{L_{\mathrm{C}}}{L_{\mathrm{Cu}}} \frac{A_{\mathrm{Cu}}}{A_{\mathrm{C}}}\right)}{\ln \frac{A_{\mathrm{Cu}}}{A_{\mathrm{C}}}}$ with atomic masses $A_{\mathrm{C}}, A_{\mathrm{Cu}}$, the thicknesses $L_{\mathrm{C}}, L_{\mathrm{Cu}}$, and densities $\rho_{\mathrm{C}}, \rho_{\mathrm{Cu}}$ as shown in Table 1 , and $N_{\mathrm{C}}, N_{\mathrm{Cu}}$ being the number of acceptance corrected events observed in the different target materials. This expression for $\alpha$ emphasizes the measurement issues. The first term depends on the corrected number of events from each target. This means that the acceptance and trigger efficiency for each different target have to be understood as a function of the kinematic variables $x_{F}$ and $p_{t}$. The second term illustrates the requirement for precision in establishing the parameters of each target used in the measurement. Any uncertainties in density or thickness translate directly into uncertainty or systematic shift in $\alpha$.

In this analysis, we reconstructed completely charm particles in specific decay modes. For $D^{0} \rightarrow K^{-} \pi^{+}, D^{0} \rightarrow$ $K^{-} \pi^{+} \pi^{+} \pi^{-}, D^{+} \rightarrow K^{-} \pi^{+} \pi^{+}, D_{s}^{+} \rightarrow K^{-} K^{+} \pi^{+}$, $\Lambda_{c}^{+} \rightarrow p K^{-} \pi^{+}$, and the corresponding charge-conjugated modes, we used cuts similar to those in previous publications [24-26]. Secondary vertex reconstruction was attempted when the $\chi^{2}$ per degree of freedom for the fit of the ensemble of charged tracks to a single primary vertex exceeded 4. All combinations of tracks were formed for secondary vertices $\left(\chi_{\mathrm{sec}}^{2}<5\right)$ and tested against a reconstruction table that specified selection criteria for each charm decay mode. Secondary vertices which occurred inside the volume of a target were rejected. The resolution of the primary vertex position is on average better than $300 \mu \mathrm{m}$ (de- 
pending slightly on the target foil), less than the thickness of the target foils and much less than the spacing between foils. This permits an unambiguous assignment of the interaction to a specific target foil. Additional identification criteria for the different decay modes required that proton and kaon candidate tracks were identified by the RICH detector to be at least as likely as a pion. Additionally, in the case of $D_{s}^{ \pm} \rightarrow K^{+} K^{-} \pi^{ \pm}$for the kaon tracks the kaon hypothesis had to be more likely than the proton hypothesis. If a pion candidate track reached the RICH detector, we applied as a loose requirement that it had to have a likelihood of at least $10 \%$; if the track failed to reach the $\mathrm{RICH}$, the candidate was called a pion. The separation between the primary and secondary vertices had to be greater than eight times its uncertainty, and the uncertainty itself less than $0.17 \mathrm{~cm}$; the reconstructed charm momentum vector had to point back to the primary vertex, and two of the daughter tracks had to have a miss distance with respect to the primary vertex of more than $\sqrt{6}$ times its uncertainty. For $D^{\star}$ states decaying into $D^{0} \pi^{ \pm}$, we required a reconstructed $D^{0}$ within $\pm 36 \mathrm{MeV} / c^{2}$ ( \pm 3 times the resolution) of the nominal mass, and an additional pion from the primary vertex. The approximate total yields for the different modes and beam particles are shown in Table 2. The invariant mass distributions were divided into groups for the primary interaction happening in one of the target foils, and further in different $x_{F}$-bins, and in some cases also in bins of $p_{t}^{2}$. We used the sidebandsubtraction technique to remove the background from the mass distributions. The resultant 736 different yields are the primary data for measuring $\alpha$.

The total acceptance (geometrical acceptance and reconstruction efficiencies) for the different decay modes of interest was estimated by embedding Monte Carlo charm decay tracks into data events. Events were generated with independently-parametrized transverse and longitudinal momentum distributions, tuned to match the shapes of the data. Detector hits, including resolution and multiple Coulomb scattering smearing effects, produced by these embedded tracks were folded into arrays of hits from real events. The new ensemble of hits was passed through the SELEX off-line software. We verified that the acceptance and reconstruction efficiency does not depend on the multiplicity of the underlying event; additionally we found that the multiplicity distributions are nearly independent of the target material, as shown in Fig. 2. The acceptance is the ratio of the number of reconstructed events over the number of embedded events in a particular mode for a specific target foil and bin in $x_{F}$ and $p_{t}^{2}$. As seen from (1), most acceptance, trigger and filter effects cancel in this measurement, only differences in the acceptances between the target foils are important; the largest effects depend on the lifetime of the different states. For example, for $\Lambda_{c}^{+}$decays in the $x_{F}$ interval $0.4-0.6$ the acceptance varies from $21.4 \%$ to $23.5 \%$ between target 7 and target 10 , while for $D^{+}$decays the variation is from $41.4 \%$ to $31.8 \%$. We verified our acceptance corrections by comparing the corrected event yields as functions of $x_{F}$ and $p_{t}$ for our three identical diamond targets and found good agreement within our statistics. This study was performed for all the charm decay modes reported here, as well as for the high statistics sample $\Lambda^{0} \rightarrow p \pi^{-}$.

For the determination of $\alpha$ we use the number of observed events, corrected for acceptances, from the three diamond targets, but only from the second copper target (target number 7). Some fraction of the events with the interaction in target number 6 where vetoed in the hardware trigger by the "S4" scintillation counter due to back-splash from the
Table 2 Raw yields (before applying any acceptance corrections) for the charm particles and modes, for the different beam particles, used in this analysis. These yields were obtained fitting a Gaussian and a polynomial representing the background to the invariant mass distributions

\begin{tabular}{|c|c|c|c|c|c|}
\hline & \multirow[t]{2}{*}{ Decay Mode } & \multicolumn{4}{|c|}{ Beam Particle } \\
\hline & & $\overline{\Sigma^{-}}$ & $\pi^{-}$ & $p$ & $\pi^{+}$ \\
\hline 1 & $D^{0} \rightarrow K^{-} \pi^{+}$ & $1176 \pm 38$ & $411 \pm 22$ & $245 \pm 16$ & $29 \pm 7$ \\
\hline 2 & $\overline{D^{0}} \rightarrow K^{+} \pi^{-}$ & $1740 \pm 52$ & $452 \pm 23$ & $437 \pm 24$ & $39 \pm 7$ \\
\hline 3 & $D^{0} \rightarrow K^{-} \pi^{+} \pi^{+} \pi^{-}$ & $1282 \pm 50$ & $467 \pm 26$ & $252 \pm 18$ & $47 \pm 6$ \\
\hline 4 & $\overline{D^{0}} \rightarrow K^{+} \pi^{-} \pi^{+} \pi^{-}$ & $1650 \pm 60$ & $488 \pm 29$ & $331 \pm 26$ & $73 \pm 9$ \\
\hline 5 & $D^{+} \rightarrow K^{-} \pi^{+} \pi^{+}$ & $1352 \pm 46$ & $361 \pm 23$ & $248 \pm 20$ & $42 \pm 7$ \\
\hline 6 & $D^{-} \rightarrow K^{+} \pi^{-} \pi^{-}$ & $2024 \pm 58$ & $555 \pm 27$ & $338 \pm 22$ & $56 \pm 9$ \\
\hline 7 & $D^{*+} \rightarrow D^{0}\left(K^{-} \pi^{+}\right) \pi^{+}$ & $165 \pm 13$ & $48 \pm 7$ & $33 \pm 7$ & - \\
\hline 8 & $D^{*-} \rightarrow \overline{D^{0}}\left(K^{+} \pi^{-}\right) \pi^{-}$ & $331 \pm 20$ & $70 \pm 8$ & $65 \pm 8$ & - \\
\hline 9 & $D^{*+} \rightarrow D^{0}\left(K^{-} \pi^{+} \pi^{+} \pi^{-}\right) \pi^{+}$ & $235 \pm 15$ & $61 \pm 9$ & $58 \pm 9$ & - \\
\hline 10 & $D^{*-} \rightarrow \overline{D^{0}}\left(K^{+} \pi^{-} \pi^{+} \pi^{-}\right) \pi^{-}$ & $446 \pm 21$ & $116 \pm 11$ & $80 \pm 10$ & - \\
\hline 11 & $D_{s}^{+} \rightarrow K^{-} K^{+} \pi^{+}$ & $118 \pm 17$ & $62 \pm 11$ & - & - \\
\hline 12 & $D_{s}^{-} \rightarrow K^{+} K^{-} \pi^{-}$ & $379 \pm 26$ & $91 \pm 12$ & - & - \\
\hline 13 & $\Lambda_{c}^{+} \rightarrow p K^{-} \pi^{+}$ & $1130 \pm 39$ & $172 \pm 15$ & $240 \pm 16$ & - \\
\hline 14 & $\overline{\Lambda_{c}^{-}} \rightarrow \bar{p} K^{+} \pi^{-}$ & $313 \pm 34$ & $95 \pm 13$ & $42 \pm 9$ & - \\
\hline
\end{tabular}




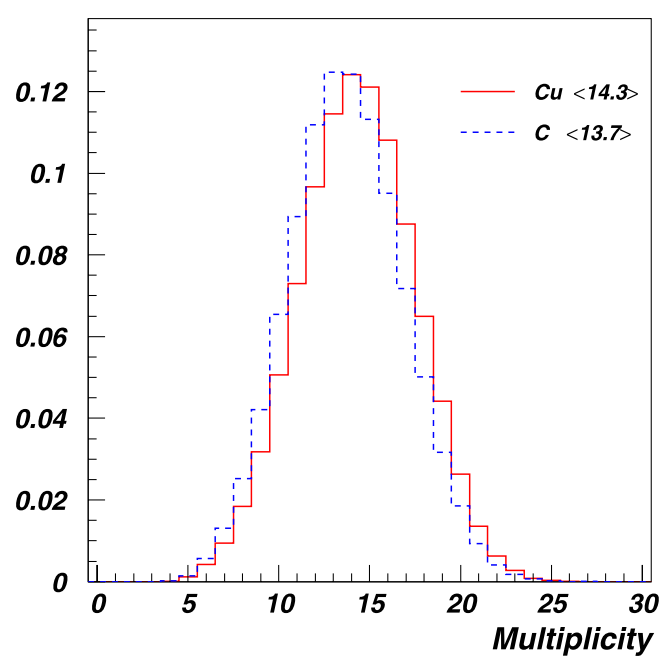

Fig. 2 Multiplicity distributions (number of tracks in the vertex region) for events with charm candidates used in this analysis, for copper (full red) and carbon (dashed blue) targets. The corresponding averages are also shown

interaction. In the attempt to correct for this vetoing we encountered systematic biases which would increase the combined statistical and systematic uncertainties more than if we ignore completely the interactions from the first copper target foil. Nevertheless, the results using target 6 are consistent with those without it.

\section{Results}

For any single mode, for four different beam particles, we calculated $\alpha$ according to (1). The results are presented

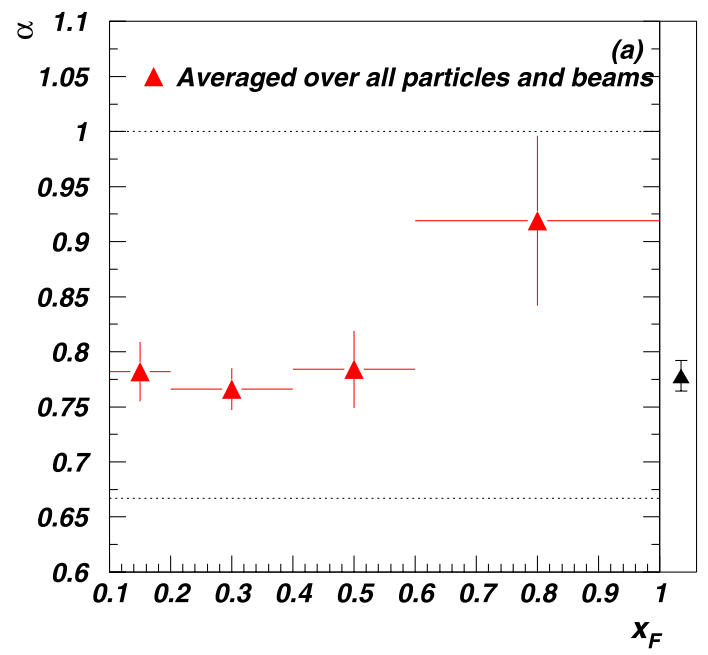

Fig. 3 Average $\alpha$ as function of $x_{F}$ for all observed final states (a) and for charm and anti-charm (b). The data points are slightly offset to avoid overlapping of the error bars. Reference $\alpha$ values of $2 / 3$ and in Table 3. Since any single measurement has a large statistical uncertainty, we average our results, weighted by the individual uncertainties, for different groups of interest.

In Fig. 3(a) we show $\alpha$ as a function of $x_{F}$ for all data, i.e., averaged over all charm and anti-charm modes and all beam particles. In Fig. 3(b) we separate the charm and anti-charm final states and show $\alpha$ averaged over all decay modes and beam particles for each type of charm quark.

In Fig. 4(a) we display the dependence of the average $\alpha$ on the beam particle type: meson or baryon. All charm and anti-charm decay modes are averaged. In Fig. 4(b, c) we separate the charm or anti-charm decays into leading and non-leading classes. Recall that leading charm processes are those in which the produced charm hadron carries at least one valence quark of the beam particle. Non-leading charm processes have no valence quarks in common between the beam and charm hadrons. In Fig. 4(c) we exclude the $D^{0}$ and $\overline{D^{0}}$ as these states could be produced preferably via excited states with different assignments to the leading/non-leading groups.

In Fig. 5(a) we present the dependence of $\alpha$ on $x_{F}$ for low $p_{t}^{2}$ and high $p_{t}^{2}$ events for $\Lambda_{c}^{+}$production by $\Sigma^{-}$beam events, to look for possible intrinsic charm effects [27]. Measurements of $\alpha$ are also available for $D$-meson production from the $\pi^{-} N$ experiments WA82 [11] ( $\mathrm{Si}, \mathrm{Cu}, \mathrm{W}$ targets), E769 [12] (Be, Cu, Al, W), and WA92 [15] (Cu, W). We compare our $D$-meson results from the $\pi^{-}$data to those from the other experiments in Fig. 5(b).

As a systematic check, we performed the identical analysis with $\Lambda^{0}$ and found good agreement with previous measurements $[7,28]$ for both proton and $\Sigma^{-}$beams. Details

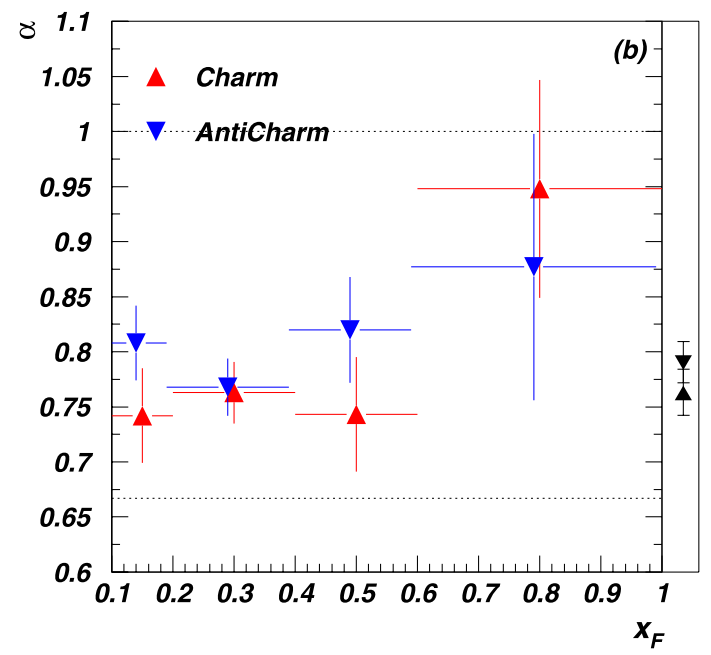

1 are shown as dotted lines. The points at $x_{F}>1$ show the average assuming that $\alpha$ does not depend on $x_{F}$ 
Table $3 \alpha$-values for the different charm particles and decay modes, for different beam particles. Only statistical uncertainties are shown. We do not determine $\alpha$ for a specific mode and/or bin of $x_{F}$ if the number of observed events (after sideband-subtraction) is below 1

\begin{tabular}{|c|c|c|c|c|c|}
\hline Beam & Mode & $\begin{array}{l}\alpha \\
0.1<x_{F}<0.2\end{array}$ & $\begin{array}{l}\alpha \\
0.2<x_{F}<0.4\end{array}$ & $\begin{array}{l}\alpha \\
0.4<x_{F}<0.6\end{array}$ & $\begin{array}{l}\alpha \\
x_{F}>0.6\end{array}$ \\
\hline$\Sigma^{-}$ & 1 & $0.75 \pm 0.07$ & $0.72 \pm 0.07$ & $0.48 \pm 0.25$ & - \\
\hline$\Sigma^{-}$ & 2 & $0.80 \pm 0.05$ & $0.70 \pm 0.06$ & $0.98 \pm 0.18$ & $0.71 \pm 1.54$ \\
\hline$\Sigma^{-}$ & 3 & $0.52 \pm 0.18$ & $0.66 \pm 0.09$ & $0.57 \pm 0.22$ & $0.67 \pm 0.68$ \\
\hline$\Sigma^{-}$ & 4 & $0.47 \pm 0.19$ & $0.67 \pm 0.09$ & $0.80 \pm 0.17$ & $1.23 \pm 0.92$ \\
\hline$\Sigma^{-}$ & 5 & $0.75 \pm 0.09$ & $0.68 \pm 0.07$ & $0.33 \pm 0.27$ & - \\
\hline$\Sigma^{-}$ & 6 & $0.80 \pm 0.08$ & $0.79 \pm 0.06$ & $0.74 \pm 0.13$ & $0.84 \pm 0.59$ \\
\hline$\Sigma^{-}$ & 7 & $0.86 \pm 0.24$ & $0.89 \pm 0.15$ & $0.57 \pm 0.31$ & - \\
\hline$\Sigma^{-}$ & 8 & $0.63 \pm 0.19$ & $0.73 \pm 0.11$ & $0.74 \pm 0.20$ & - \\
\hline$\Sigma^{-}$ & 9 & $0.43 \pm 0.45$ & $0.41 \pm 0.17$ & $0.88 \pm 0.17$ & - \\
\hline$\Sigma^{-}$ & 10 & $0.80 \pm 0.21$ & $0.80 \pm 0.10$ & $0.84 \pm 0.14$ & $0.47 \pm 0.50$ \\
\hline$\Sigma^{-}$ & 11 & $1.10 \pm 0.38$ & $1.07 \pm 0.19$ & - & - \\
\hline$\Sigma^{-}$ & 12 & $0.99 \pm 0.35$ & $0.79 \pm 0.12$ & $0.87 \pm 0.16$ & - \\
\hline$\Sigma^{-}$ & 13 & $0.70 \pm 0.20$ & $0.95 \pm 0.08$ & $0.90 \pm 0.10$ & $0.83 \pm 0.17$ \\
\hline$\Sigma^{-}$ & 14 & $1.32 \pm 0.25$ & $0.74 \pm 0.24$ & - & - \\
\hline$\pi^{-}$ & 1 & $0.86 \pm 0.14$ & $0.82 \pm 0.11$ & $0.25 \pm 0.24$ & - \\
\hline$\pi^{-}$ & 2 & $0.89 \pm 0.12$ & $0.78 \pm 0.11$ & $0.96 \pm 0.17$ & - \\
\hline$\pi^{-}$ & 3 & $0.85 \pm 0.32$ & $0.87 \pm 0.12$ & $0.82 \pm 0.17$ & $1.00 \pm 0.18$ \\
\hline$\pi^{-}$ & 4 & $1.04 \pm 0.22$ & $0.88 \pm 0.15$ & $0.59 \pm 0.19$ & $0.75 \pm 0.29$ \\
\hline$\pi^{-}$ & 5 & $0.37 \pm 0.34$ & $0.70 \pm 0.13$ & $0.79 \pm 0.17$ & $1.27 \pm 0.37$ \\
\hline$\pi^{-}$ & 6 & $0.76 \pm 0.18$ & $0.75 \pm 0.10$ & $0.89 \pm 0.16$ & $0.83 \pm 0.21$ \\
\hline$\pi^{-}$ & 7 & $1.50 \pm 0.56$ & $1.17 \pm 0.33$ & $0.68 \pm 0.36$ & - \\
\hline$\pi^{-}$ & 8 & $0.34 \pm 1.06$ & $1.11 \pm 0.24$ & $0.77 \pm 0.32$ & - \\
\hline$\pi^{-}$ & 9 & $1.54 \pm 0.60$ & $0.95 \pm 0.25$ & $0.29 \pm 0.46$ & $1.00 \pm 0.38$ \\
\hline$\pi^{-}$ & 10 & - & $0.99 \pm 0.22$ & $0.70 \pm 0.25$ & $1.01 \pm 0.22$ \\
\hline$\pi^{-}$ & 11 & - & $0.42 \pm 0.49$ & $0.83 \pm 0.58$ & - \\
\hline$\pi^{-}$ & 12 & - & $0.87 \pm 0.24$ & $0.68 \pm 0.44$ & $1.52 \pm 1.04$ \\
\hline$\pi^{-}$ & 13 & $1.42 \pm 0.54$ & $1.08 \pm 0.18$ & $0.84 \pm 0.27$ & $0.80 \pm 0.37$ \\
\hline$\pi^{-}$ & 14 & - & - & $0.95 \pm 0.37$ & - \\
\hline$p$ & 1 & $0.56 \pm 0.18$ & $0.65 \pm 0.14$ & $0.76 \pm 0.56$ & - \\
\hline$p$ & 2 & $0.77 \pm 0.12$ & $0.67 \pm 0.12$ & $0.32 \pm 0.41$ & - \\
\hline$p$ & 3 & $0.77 \pm 0.34$ & $0.53 \pm 0.20$ & $0.74 \pm 0.27$ & - \\
\hline$p$ & 4 & $0.61 \pm 0.35$ & $0.45 \pm 0.20$ & $0.37 \pm 0.54$ & $0.93 \pm 2.32$ \\
\hline$p$ & 5 & $0.50 \pm 0.26$ & $0.71 \pm 0.14$ & - & - \\
\hline$p$ & 6 & $0.94 \pm 0.18$ & $0.80 \pm 0.12$ & $1.02 \pm 0.26$ & - \\
\hline$p$ & 7 & - & $0.61 \pm 0.35$ & $0.47 \pm 0.66$ & - \\
\hline$p$ & 8 & - & $1.03 \pm 0.19$ & $0.35 \pm 0.65$ & - \\
\hline$p$ & 9 & - & - & $0.48 \pm 0.48$ & - \\
\hline$p$ & 10 & - & $0.73 \pm 0.23$ & $0.22 \pm 0.46$ & - \\
\hline$p$ & 13 & $0.51 \pm 0.77$ & $0.44 \pm 0.23$ & $0.79 \pm 0.22$ & $1.03 \pm 0.30$ \\
\hline$p$ & 14 & - & $1.08 \pm 0.80$ & $0.78 \pm 0.55$ & - \\
\hline$\pi^{+}$ & 1 & $0.37 \pm 0.65$ & $1.08 \pm 0.29$ & $0.23 \pm 0.65$ & - \\
\hline$\pi^{+}$ & 2 & $0.68 \pm 0.34$ & $1.23 \pm 0.22$ & $0.38 \pm 0.68$ & - \\
\hline$\pi^{+}$ & 3 & - & $1.00 \pm 0.41$ & $0.91 \pm 0.35$ & $1.15 \pm 0.88$ \\
\hline$\pi^{+}$ & 4 & - & $0.74 \pm 0.32$ & $1.57 \pm 0.51$ & - \\
\hline$\pi^{+}$ & 5 & $1.11 \pm 0.67$ & $0.43 \pm 0.38$ & $0.58 \pm 0.80$ & - \\
\hline$\pi^{+}$ & 6 & $0.59 \pm 0.76$ & $0.90 \pm 0.25$ & $1.28 \pm 0.33$ & $1.03 \pm 0.60$ \\
\hline
\end{tabular}



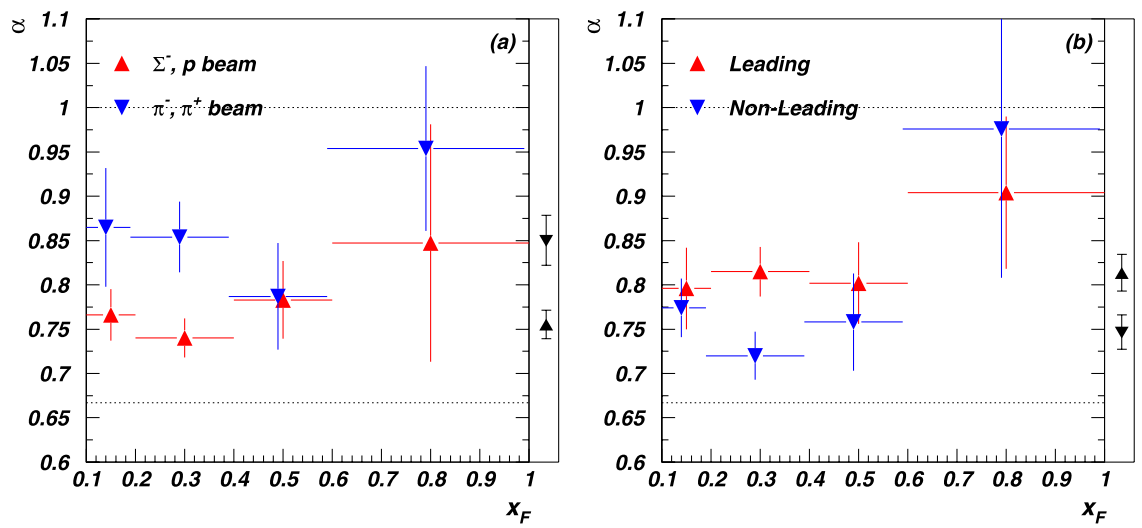

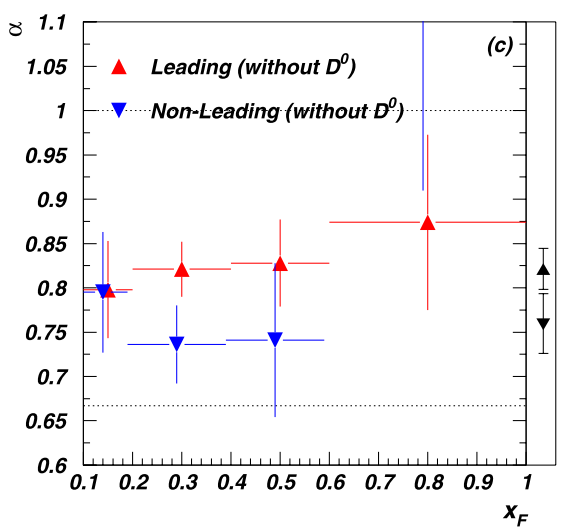

Fig. 4 Average $\alpha$ as a function of $x_{F}$ for production by baryon $\left(\Sigma^{-}\right.$, $p$ ) and meson $\left(\pi^{ \pm}\right)$beams (a) and leading and non-leading (b, c) particles. The data points are slightly offset to avoid overlapping of the error

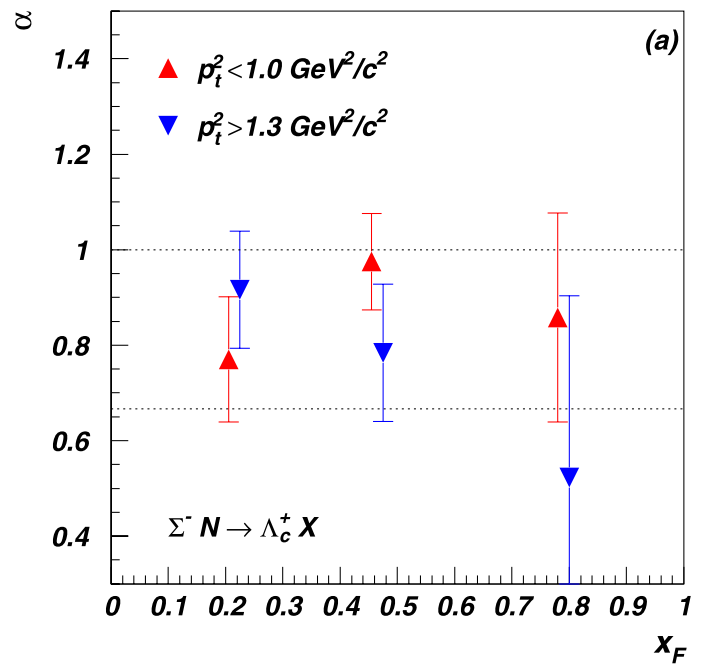

Fig. $5 \alpha$ for the production of $\Lambda_{c}^{+}$with a $\Sigma^{-}$(a) and for $D^{ \pm}$, $D^{0}$ mesons with $\pi^{-}$(b) beam, as function of $x_{F}$ for low $\left(p_{t}^{2}<\right.$ $\left.1.0 \mathrm{GeV}^{2} / c^{2}\right)$ and high $\left(p_{t}^{2}>1.3 \mathrm{GeV}^{2} / c^{2}\right)$ transverse momentum. Our data points are slightly offset to avoid overlapping of the error

will be presented in a forthcoming publication [29]. We looked for variations of $\alpha$ with any of the event selection cuts. All changes were small compared with the statistical uncertainty, indicating negligible systematic error from the cut selections. We also studied binning effects and found only small shifts, compatible with statistical uncertainties only.

\section{Discussion and conclusions}

As seen from the figures, all the measured values are compatible with being independent of $x_{F}$. Averaging over all our data, we obtain $\alpha=0.778 \pm 0.014$, which is incompatible with both usually suggested values of $2 / 3$ and 1 . Additional bars. Reference $\alpha$ values of $2 / 3$ and 1 are shown as dotted lines. The points at $x_{F}>1$ show the average assuming that $\alpha$ does not depend on $x_{F}$

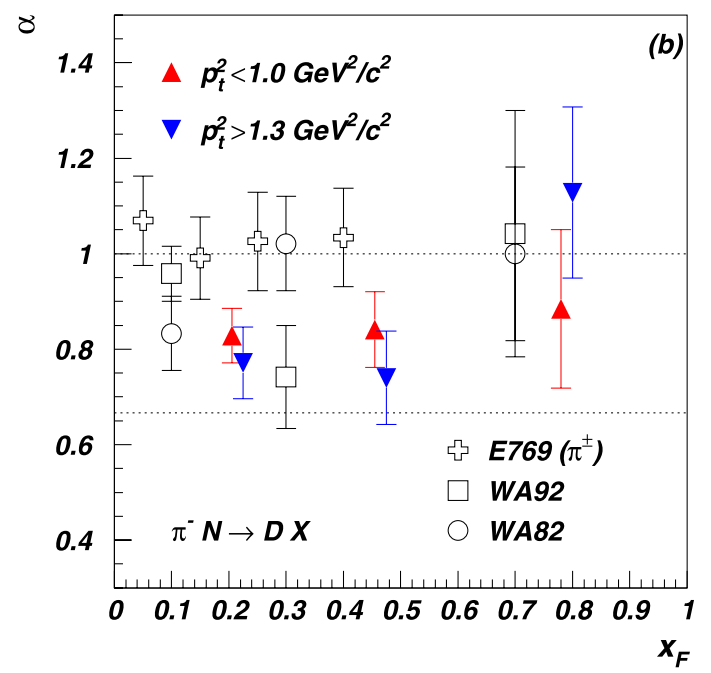

bars. Reference $\alpha$ values of $2 / 3$ and 1 are shown as dotted lines. Also shown (open symbols) are results $[11,12,15]$ from other experiments, without separation in $p_{t}^{2}$

averages for different groupings are show in Table 4. Averaging separately over charm and anti-charm final states the $\alpha$ values show no difference. Separating into leading and nonleading production (Fig. 4(b)) we obtain a $2.3 \sigma$ difference, which vanishes due to the increased uncertainties when we exclude the $D^{0}$ 's in this comparison (Fig. 4(c)). Separating the data into production by meson beams and that by baryon beams (Fig. 4(a)), there is a difference in the $\alpha$ value averaged over all charm and anti-charm modes, corresponding to a $3 \sigma$ effect.

For production of $\Lambda_{c}^{+}$particles with a $\Sigma^{-}$beam the behavior shown in Fig. 5(a) seems to suggest a decrease for high $x_{F}$ and $p_{t}^{2}$, compared to $D$-mesons results shown in Fig. 5(b). We note that $D$-meson data has only a small contribution from events with $p_{t}^{2}>1.3 \mathrm{GeV}^{2} / c^{2}$, so no firm 
Table $4 \alpha$ values for various grouping of charm species and/or beams

\begin{tabular}{ll}
\hline Average over $x_{F}$ and & $\alpha$ \\
\hline All species and all beams & $0.778 \pm 0.014$ \\
All species for meson beams & $0.850 \pm 0.028$ \\
All species for baryon beams & $0.755 \pm 0.016$ \\
All beams for charm particles & $0.763 \pm 0.021$ \\
All beams for anti-charm particles & $0.791 \pm 0.019$ \\
All beams for leading particles & $0.814 \pm 0.021$ \\
$\quad$ Same as above, but excluding $D^{0}, \mathrm{~s}$ & $0.821 \pm 0.023$ \\
All beams for non-leading particles & $0.747 \pm 0.019$ \\
$\quad$ Same as above, but excluding $D^{0}, \mathrm{~s}$ & $0.760 \pm 0.034$ \\
$\Lambda_{c}^{+}$for $\Sigma^{-}$beam, $p_{t}^{2}<1.0 \mathrm{GeV}^{2} / c^{2}$ & $0.894 \pm 0.075$ \\
$\Lambda_{c}^{+}$for $\Sigma^{-}$beam, $p_{t}^{2}>1.3 \mathrm{GeV}^{2} / c^{2}$ & $0.841 \pm 0.091$ \\
$D$ mesons for $\pi^{-}$beam, $p_{t}^{2}<1.0 \mathrm{GeV}^{2} / c^{2}$ & $0.836 \pm 0.045$ \\
$D$ mesons for $\pi^{-}$beam, $p_{t}^{2}>1.3 \mathrm{GeV}^{2} / c^{2}$ & $0.796 \pm 0.057$ \\
\hline
\end{tabular}

conclusion can be drawn. All distributions are consistent with no dependence on $x_{F}$.

In summary, within our statistics there is no dependence of $\alpha$ on $x_{F}$ for any charm species for the interval $0.1<x_{F}<$ 1.0. The average value of $\alpha$ for charm production by pion beams is $\alpha_{\text {meson }}=0.850 \pm 0.028$. This is somewhat larger than the corresponding average $\alpha_{\text {baryon }}=0.755 \pm 0.016$ for charm production by baryon beams $\left(\Sigma^{-}, p\right)$.

Acknowledgements We thank S. Brodsky (SLAC) for useful discussions. The authors are indebted to the staff of Fermi National Accelerator Laboratory and for invaluable technical support from the staffs of collaborating institutions. This project was supported in part by Bundesministerium für Bildung, Wissenschaft, Forschung und Technologie, Consejo Nacional de Ciencia y Tecnología (CONACyT), Conselho Nacional de Desenvolvimento Científico e Tecnológico, Fondo de Apoyo a la Investigación (UASLP), Fundação de Amparo à Pesquisa do Estado de São Paulo (FAPESP), the Israel Science Foundation founded by the Israel Academy of Sciences and Humanities, Istituto Nazionale di Fisica Nucleare (INFN), the International Science Foundation (ISF), the National Science Foundation (Phy \#9602178), NATO (grant CR6.941058-1360/94), the Russian Academy of Science, the Russian Ministry of Science and Technology, the Russian Foundation for Basic Research (grant 08-02-00657), the Secretaría de Educación Pública (Mexico) (grant number 2003-24-001-026), the Turkish Scientific and Technological Research Board (TÜBİTAK), and the U.S. Department of Energy (DOE grant DE-FG02-91ER40664 and DOE contract number DE-AC02-76CHO3000).

\section{References}

1. H. Cobbaert et al. (WA78 Collaboration), Phys. Lett. B 191, 456 (1987)
2. H. Cobbaert et al. (WA78 Collaboration), Phys. Lett. B 206, 546 (1988)

3. H. Cobbaert et al. (WA78 Collaboration), Phys. Lett. B 213, 395 (1988)

4. M.J. Leitch et al. (FNAL E866/NuSea Collaboration), Phys. Rev. Lett. 84, 3256 (2000). arXiv:nucl-ex/9909007

5. B. Alessandro et al. (NA50 Collaboration), Eur. Phys. J. C 33, 31 (2004)

6. I. Abt et al. (HERA-B Collaboration), Eur. Phys. J. C 60, 525 (2009). arXiv:0812.0734 [hep-ex]

7. K.J. Heller et al., Phys. Rev. D 16, 2737 (1977)

8. P. Skubic et al., Phys. Rev. D 18, 3115 (1978)

9. A.N. Aleev et al. (BIS-2 Collaboration), Sov. J. Nucl. Phys. 46, 657 (1987). [Yad. Fiz. 46, 1127 (1987)]

10. M. Vecko et al. (BIS-2 Collaboration), Czech. J. Phys. B 39, 297 (1989)

11. M. Adamovich et al. (WA82 Collaboration), Phys. Lett. B 284, 453 (1992)

12. G.A. Alves et al. (Fermilab E769 Collaboration), Phys. Rev. Lett. 70, 722 (1993)

13. G.A. Alves et al. (E769 Collaboration), Phys. Rev. D 49, 4317 (1994)

14. M.J. Leitch et al. (E789 Collaboration), Phys. Rev. Lett. 72, 2542 (1994)

15. M. Adamovich et al. (BEATRICE Collaboration), Nucl. Phys. B 495, 3 (1997)

16. L. Apanasevich et al. (Fermilab E706 Collaboration), Phys. Rev. D 56, 1391 (1997). arXiv:hep-ex/9702014

17. I. Abt et al. (HERA-B Collaboration), Eur. Phys. J. C 52, 531 (2007). arXiv:0708.1443 [hep-ex]

18. M.E. Duffy et al., Phys. Rev. Lett. 55, 1816 (1985)

19. R. Vogt (Hard Probe Collaboration), Int. J. Mod. Phys. E 12, 211 (2003). arXiv:hep-ph/0111271

20. C. Lourenco, H.K. Wohri, Phys. Rept. 433, 127 (2006). arXiv:hep-ph/0609101

21. A.D. Frawley, T. Ullrich, R. Vogt, Phys. Rept. 462, 125 (2008). arXiv:0806.1013 [nucl-ex]

22. J.S. Russ et al. (SELEX Collaboration), in Proceedings of the 29th International Conference on High Energy Physics, ed. by A. Astbury et al., vol. II (World Scientific, Singapore, 1998), p. 1259. arXiv:hep-ex/9812031

23. J. Engelfried et al., Nucl. Instrum. Methods A 431, 53 (1999). arXiv:hep-ex/9811001

24. A. Kushnirenko et al. (SELEX Collaboration), Phys. Rev. Lett. 86, 5243 (2001). arXiv:hep-ex/0010014

25. F.G. Garcia et al. (SELEX Collaboration), Phys. Lett. B 528, 49 (2002). arXiv:hep-ex/0109017

26. M. Kaya et al. (SELEX Collaboration), Phys. Lett. B 558, 34 (2003). arXiv:hep-ex/0302039

27. S.J. Brodsky, B. Kopeliovich, I. Schmidt, J. Soffer, Phys. Rev. D 73, 113005 (2006). arXiv:hep-ph/0603238

28. M.I. Adamovich et al. (WA89 Collaboration), Eur. Phys. J. C 26, 357 (2003)

29. A. Blanco-Covarrubias et al. (SELEX Collaboration), in preparation 